\title{
Development of HPLC Determination Method for Trace Levels of 1-, 2-Nitropyrenes and 2-Nitrofluoranthene in Airborne Particulates and Its Application to Samples Collected at Noto Peninsula
}

\author{
Kazuichi Hayakawa*, Ning Tang, Kosuke Sato, Akihiko Izaki, Michiya Tatematsu, Hirotaka Hama, \\ Ying Li, Takayuki Kameda and Akira Toriba \\ Institute of Medical, Pharmaceutical and Health Sciences, Kanazawa University, Kakuma-machi, Kanazawa 920-1192, Japan
}

*Corresponding author. Tel: +81-76-234-4413, E-mail: hayakawa@p.kanazawa-u.ac.jp

\begin{abstract}
1-Nitropyrene (1-NP), 2-NP and 2-nitrofluoranthene (2-NFR) are useful markers for studying the atmospheric behaviors of polycyclic aromatic hydrocarbons (PAHs) and nitropolycyclic aromatic hydrocarbons (NPAHs). However, present methods for measuring trace levels of these compounds are lesssensitive and laborious. Here we describe several improvements to a previously reported high-performance liquid chromatography-chemiluminescence detection system that allows it to determine trace levels of 1-, 2-NPs and 2-NFR. The proposed system was equipped with a reducer column packed with $\mathrm{Pt} / \mathrm{Rh}$ instead of zinc whose life-time was limited. The combination of Cosmosil MS-II (monomeric ODS) and AR-II (polymeric ODS) columns was used instead of polymeric ODS columns as the separator column to improve the separation. An ethanol mixture with acetate buffer ( $\mathrm{pH}$ 5.5) was used in place of an acetonitrile mixture with the same buffer to activate the reducer column. The same ethanol mixture was used as the mobile phase for the clean-up column. The switching time of the column switching valve was optimized to concentrate the amino-derivatives of above NPAHs quantitatively on the concentrator column. The concentrations of bis(2,4,6-trichlorophenly) oxalate and hydrogen peroxide in the chemiluminescence reagent solution were optimized to $0.4 \mathrm{mM}$ and $30 \mathrm{mM}$, respectively, to increase the sensitivity. Under the above conditions, the detection limits (S/N $=3$ ) of 1 -, 2-NPs and 2-NFR were $1 \mathrm{fmol}(0.25 \mathrm{pg})$, $10 \mathrm{fmol}(2.5 \mathrm{pg})$ and $4 \mathrm{fmol}(1 \mathrm{pg})$, respectively. The proposed system was effectively used to determine trace levels of 1-, 2-NPs and 2-NFR in airborne particulates collected at Noto Peninsula. The atmospheric concentrations of 1-, 2-NPs and 2-NFR were not more than sub $\mathrm{pg} \mathrm{m}^{-3}$ levels. They were higher in
\end{abstract}

winter (January) than in summer (July). In both seasons, the concentrations were in decreasing order, [2-NFR] $>$ [1-NP] $>$ [2-NP].

Key words: 1-Nitropyrene, 2-Nitropyrene, 2-Nitrofluoranthene, Trace analysis, Airborne particulate matter

\section{INTRODUCTION}

Among the air pollutants exhausted from the combustion of fossil fuels such as petroleum and coal, polycyclic aromatic hydrocarbons (PAHs) show carcinogenicity and/or mutagenicity (Tokiwa et al., 1980), endocrine disrupting activity (Kizu et al., 2000) or reactive oxygen species producing activity (Motoyama et al., 2009). Nitropolycyclic aromatic hydrocarbons (NPAHs) such as 1-nitropyrene (1-NP) and 1,3-dinitropyrene are also exhausted from the combustion of fossil fuels, and their mutagenicity was much stronger than that of benzo $[a]$ pyrene. Additionally, several NPAHs such as 2-NP and 2-nitrofluoranthene (2-NFR) are subsequently formed by the reaction of PAHs and NOx in the air (Arey et al., 1986), suggesting that 1-, 2-NPs and 2NFR are markers for studying the atmospheric behaviors of PAHs and NPAHs. Therefore, a method for simultaneously determining these three NPAHs would be useful. The authors developed a highly sensitive determination method for NPAHs in environmental samples such as airborne particulates and particulates exhausted from automobiles by using high-performance liquid chromatography (HPLC) with chemiluminescence detection (CLD) (Hayakawa et al., 1995, 1991; Imaizumi et al., 1990). Although NPAH concentrations in urban air were high enough to be determined by HPLC-CLD, the concentrations were much lower in the air at suburban and background sites that were not 
near any contributors such as factories or high-traffic roads. We collected one-week airborne particulates with a high-volume air sampler at Kanazawa University Wajima Air Monitoring Station in the Noto Peninsula, Japan, which was a background monitoring site. The data showed a seasonal variation of PAH concentrations caused by long-range transport from China (Yang et al., 2007). However, no NPAH were detected.

We previously determined trace levels of 1-, 2- and 4-NPs, 2-NFR and 6-nitrochrysene (6-NC) in airborne particulates and precipitation collected in urban area by introducing reducer and concentrator columns into our conventional HPLC-CLD system (Murahashi and Hayakwa, 1997). However, the life time of the reducer column packed with zinc was limited and the analysis time took more than 90 minutes since three sample injections were necessary to analyze the NPAHs. Recently, we developed an HPLC-CLD system for the simultaneous determination of more than 20 NPAHs by introducing the reducer column packed with $\mathrm{Pt} / \mathrm{Rh}$ instead of zinc, since $\mathrm{Pt} / \mathrm{Rh}$ catalyzed the reduction of NPAHs. In the system, an ethanol-aqueous solution containing ascorbic acid was used to activate the reducer column instead of an acetonitrile-aqueous solution (Tang et al., 2005, 2003; Hayakawa et al., 2001). However, the resolution and sensitivity were not high enough to detect trace levels of above NPAHs.

In this report, we improved an HPLC-CLD system with lower detection limits and higher resolutions of 1-, 2-NPs and 2-NFR by improving our previous system (Murahashi and Hayakawa, 1997). The proposed method was used for determining trace levels of 1-, 2NPs and 2-NFR in airborne particulate samples collected in winter and summer at Noto Peninsula.

\section{MATERIALS AND METHODS}

\subsection{Chemicals}

1-, 4-NPs and 2-NFR were purchased from Aldrich Company Inc. (Milwaukee, WI, U. S. A.). 2-NP was kindly supplied by Professor Akihisa Hirayama, Kyoto Pharmaceutical University. Deuterated 1-NP (1-NP- $\left.d_{9}\right)$ was purchased from CDN Isotopes Inc. (Pointe-Claire, Quebec, Canada). All other chemicals used were of analytical reagent grade.

\section{2 HPLC System and Conditions}

Three on-line reducers, including an electrochemical reactor and columns packed with metals have been reported for reducing NPAHs to their corresponding amino-derivatives (Hayakawa et al., 2001, 1993; Imaizumi et al., 1990). The electrochemical reducer showed the lowest reduction efficiency and the other two showed quantitative reduction efficiency. The life time of the zinc reducer column was limited, while the life time of the $\mathrm{Pt} / \mathrm{Rh}$ reducer column was not (Hayakawa et al., 2001, 1993). Considering these advantages of the $\mathrm{Pt} / \mathrm{Rh}$ reducer column, we set up the HPLC-CLD system with a $\mathrm{Pt} / \mathrm{Rh}$ reducer column according to our previous report (Tang et al., 2003) with several modifications (Fig. 1 ). The system consisted of four HPLC mobile phase pumps, a chemiluminescence reagent solution pump, a chemiluminescence detector, a system controller, an integrator, a degasser, two column ovens for the reducer column $\left(80^{\circ} \mathrm{C}\right)$ and for the clean-up and separator columns $\left(20^{\circ} \mathrm{C}\right)$, an auto sample injector $(100 \mu \mathrm{L})$, a switching value, a guard column, a concentrator column, a clean-up column, a reducer column $(4.0$ i.d. $\times 10 \mathrm{~mm}$, packed with $\mathrm{Pt} / \mathrm{Rh}$, Shimadzu) and two separator columns (Cosmosil 5C18-MS-II, 4.6 i.d. $\times 150 \mathrm{~mm}$, Nacalai Tesqu and 5C18-AR-II, 4.6 i.d. $\times 250 \mathrm{~mm}$, Nacalai Tesque) connected in series.

The mobile phases for the clean-up column and reducer column was ethanol-acetate buffer ( $\mathrm{pH}$ 5.5) (3:1, $\mathrm{v} / \mathrm{v}$ ) at a flow rate of $0.3 \mathrm{~mL} \mathrm{~min}^{-1}$. The mobile phase for the concentrator column was the same ethanol-acetate buffer with $30 \mathrm{mM}$ ascorbic acid at a flow rate of $1.8 \mathrm{~mL} \mathrm{~min}^{-1}$. The mobile phase for the separator columns was a mixture of acetonitrile and $10 \mathrm{mM}$ imidazole buffer (pH 7.6) $(1: 1, \mathrm{v} / \mathrm{v})$ at a flow rate of $1.0 \mathrm{~mL}$ $\min ^{-1}$. The chemiluminescence reagent solutions were acetonitrile solutions containing $0.4 \mathrm{mM}$ bis(2,4,6-trichlorophenly)oxalate (TCPO) and $30 \mathrm{mM}$ hydrogen peroxide, respectively, each cooled with ice-water. Both solutions were mixed $(1: 1, \mathrm{v} / \mathrm{v})$ in the system, and the flow rate of the mixture was $1 \mathrm{~mL} \mathrm{~min}{ }^{-1}$. Other conditions were the same as in our previous reports (Tang et al., 2005, 2003).

\section{3 Preparation of Standard Solutions}

Stock standard solutions of 1-, 2-, 4-NPs, 2-NFR and $1-\mathrm{NP}-d_{9}$ were independently prepared by dissolving each crystal in ethanol. The stock standard solutions were mixed, and then the mixture was diluted adequately with acetonitrile-water $(3: 1, \mathrm{v} / \mathrm{v})$ to give calibration curve solutions: $0.1-100 \mathrm{pmol} \mathrm{L}^{-1}$ for 2-NFR; 0.4$400 \mathrm{pmol} \mathrm{L}^{-1}$ for 2-NP; $0.05-50 \mathrm{pmol} \mathrm{L}^{-1}$ for 1-NP. 1NP- $d_{9}$ standard solution was at the concentration of $0.2 \mathrm{pmol} \mathrm{L}^{-1}$ in ethanol.

\section{4 Sampling and Sample Preparations}

Airborne particulate samples were collected at Kanazawa University Wajima Air Monitoring Station at Noto Peninsula (Nisifutamata-machi, Wajima City, Ishikawa Prefecture, Japan). Airborne particulates were collected by a high volume air sampler (AH-600, Shibata Japan) with a quartz fiber filter $(8 \times 10$ inch, 2500QAT-UP, Pallflex Products, Putnam, CT, U. S. A.) at a flow rate of $0.7 \mathrm{~m}^{3} \mathrm{~min}^{-1}$ in January and July, 2007. 
The filter was newly changed every week. Airborne particulates were collected from January 4 to 11,2008 , too. One-fourth of the filter (corresponding to $3.53 \times$ $10^{3} \mathrm{~m}^{3}$ air) was cut into small pieces in a flask and 100 $\mu \mathrm{L}$ of $1-\mathrm{NP}-d_{9}$ standard solution was added to the flask as an internal standard. Then, NPAHs were extracted ultrasonically twice with benzene/ethanol $(3: 1, \mathrm{v} / \mathrm{v})$. The solution was washed successively with sodium hydroxide, sulfuric acid solution and water. After filtering the organic solution with an HLC-DISK membrane (pore size $0.45 \mu \mathrm{m}$, Kanto Chemical Co., Tokyo, Japan), the solution was evaporated to dryness. The residue was dissolved in $1.0 \mathrm{~mL}$ of ethanol, and then an aliquot $(100 \mu \mathrm{L})$ of the solution was injected into the HPLC-CLD system.

\section{RESULTS AND DISCUSSION}

\section{1 Comparison of Separator Columns}

$1-\mathrm{NP}$ is one of the major NPAHs exhausted directly from combustion processes of fossil fuel, and both 2NFR and 2-NP are major NPAHs which are secondarily formed in the air (Arey et al., 1986). These facts suggest that the simultaneous determination of 1-, 2NPs and 2-NFR is useful for determining their atmospheric behaviors. In our previous HPLC-CLD system using the reducer column packed with $\mathrm{Pt} / \mathrm{Rh}$, however, the retention time of 2-NFR was close to that of 2-NP (Tang et al., 2005). Moreover, the retention time of 4NP was similar to the retention times of 2-NFR and 2-NP, and the concentration of 4-NP was much lower (Murahashi et al., 1999). These observations suggest that the resolution of the NPAHs should be improved, resulting in more accurate determination.

Polymeric ODS columns are known to give better resolution of PAHs than monomeric ODS columns. However, the difference of those two-type ODS columns in the separation of amino-derivatives of PAHs (APAHs) has not been examined. We compared the retentions of amino-derivatives of 1-, 2-, 4-NPs (1-, 2-, 4-aminopyrenes, 1-, 2-, 4-APs) and 2-NFR (2-aminofluoranthene, 2-AFR) on Cosmosil MS-II (monomeric ODS) and AR-II (polymeric ODS) columns by introducing them into the HPLC-CLD system as shown in Fig. 1 without the two guard columns and the clean-up column. A mixture of acetonitrile and aqueous buffer is suitable for the peroxyoxalate chemiluminescence

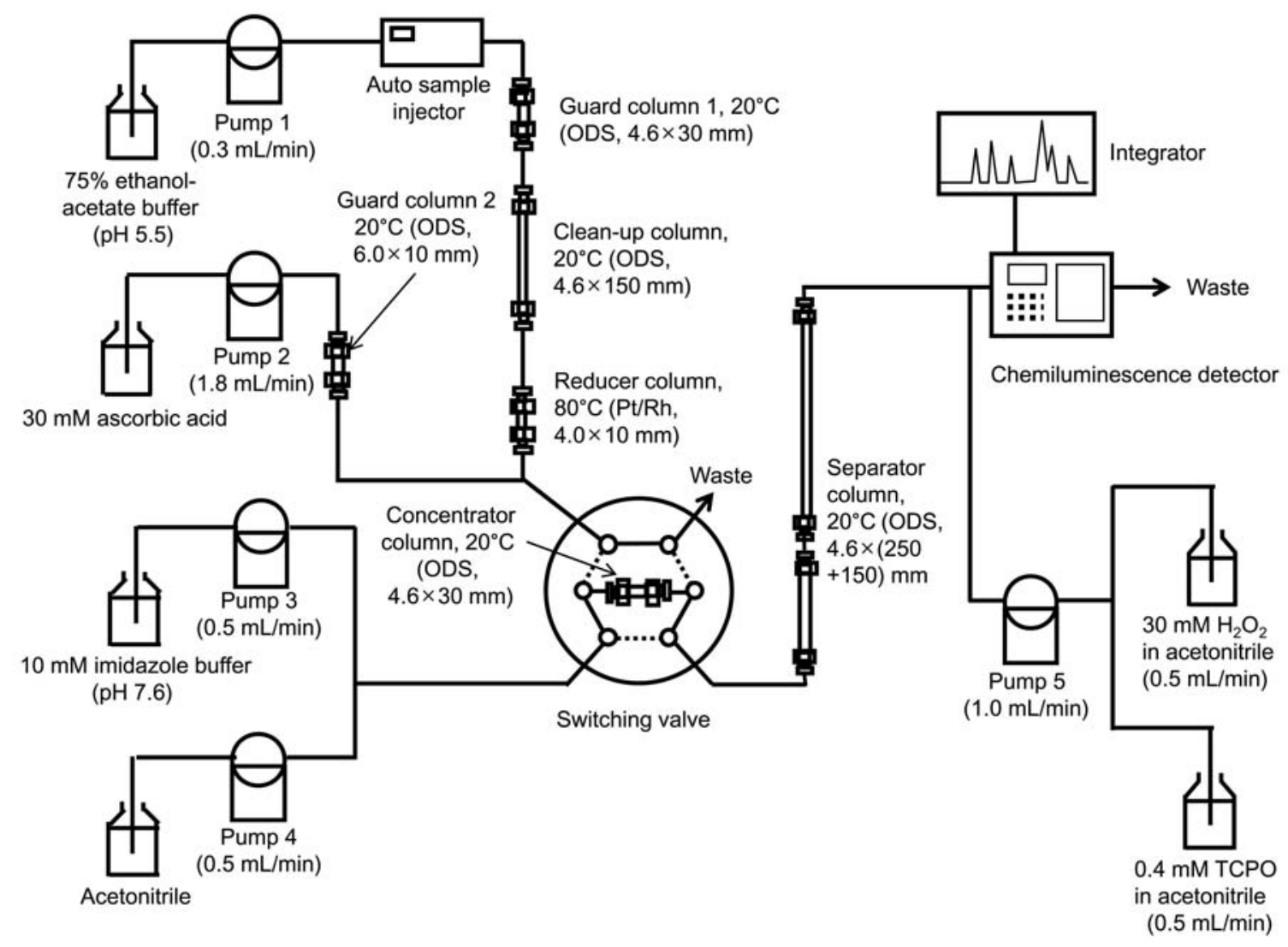

Fig. 1. Schematic diagram of the proposed HPLC-CLD system. 
Table 1. Retention times (minute) of 1-, 2-, 4-NPs and 2-NFR ${ }^{1)}$.

\begin{tabular}{cccc}
\hline Compound & MS-II $(400 \mathrm{~mm})^{2)}$ & AR-II $(400 \mathrm{~mm})^{2)}$ & MS-II $(150 \mathrm{~mm})+$ AR-II $(250 \mathrm{~mm})^{2)}$ \\
\hline 2-NFR & 48.5 & 54.5 & 52.5 \\
2-NP & 48.5 & 58 & 54.5 \\
4-NP & 51 & 58 & 57 \\
1-NP & 55.5 & 63.5 & 60.5 \\
\hline
\end{tabular}

1) 1-, 2-, 4-NPs and 2-NFR were reduced to their corresponding amino-derivatives in the reducer column and then introduced into the separator columns.

${ }^{2)}$ Inner diameter of the columns was $4.6 \mathrm{~mm}$.

detection of amino-derivatives of PAHs (Hayakawa et al., 2001, 1995). However, the reduction activity of $\mathrm{Pt} / \mathrm{Rh}$ occurs in hot ethanol but not in acetonitrile, suggesting that the carrier solution going into the reducer column must contain ethanol but not acetonitrile. Therefore, a mixture of ethanol and acetate buffer ( $\mathrm{pH} 5.5$ ) was used as the mobile phase for the reducer column. On the other hand, amino-derivatives of PAHs were unstable in the system. Ascorbic acid was added to the effluent from the reducer column to keep the aminoderivatives stable in our previous HPLC-CLD system (Murahashi and Hayakawa, 1997). Thus, ascorbic acid was added to the mobile phase for the concentrator column in the proposed system as described in Experimental.

When NPAH standard solutions were injected into the system, NPAHs were all reduced quantitatively to their corresponding amino-derivatives in the reducer column. However, the separation of 2-AFR and 2-AP was impossible on a Cosmosil MS-II column, although the separation of 1- and 2-APs was complete. On the other hand, a Cosmosil AR-II column completely separated 2-AFR and 2-AP but did not completely separate 2- and 4-APs. By using a combination of Cosmosil MS-II and AR-II columns, the four APAHs were separately determined as amino derivatives (Table 1). The mean concentration ratio of $4-\mathrm{NP}\left(0.57 \mathrm{fmol} \mathrm{m}^{-3}\right)$ was around $1 / 100$ or $1 / 6$ of those of 2-NFR and 2-NP, respectively, in the urban air of Kanazawa (Murahashi and Hayakawa, 1997), suggesting that 4-NP is not a good primary or secondary formation marker such as 1-, 2NPs and 2-NFR. Accordingly, 1-, 2-NPs and 2-NFR were selected as target compounds in the following experiments and the combination of Cosmosil MS-II column (4.6 i.d. $\times 150 \mathrm{~mm})+$ Cosmosil AR-II column $(4.6$ i.d. $\times 250 \mathrm{~mm}$ ) was used for the following experiments.

\section{2 Optimum Composition of Chemiluminescence Reagent Solution}

Next, we examined the optimal time for switching the switching valve after loading APAHs on the concentrator column of the HPLC-CLD system as shown in Fig. 1. When the standard mixture of 1-, 2-NPs, 2-
NFR and 1-NP- $d_{9}$ was injected into the system, they were eluted from the reducer column in the period from 17 to 29 minutes with the following elution order; 1 $\mathrm{NP}-d_{9}=1-\mathrm{NP}<2-\mathrm{NFR}<2-\mathrm{NP}$. From this result, the concentration time (switching time of the switching valve) was set at 17-29 minutes.

The optimum composition of chemiluminescence reagent solution is known to be different for different analytes, and the chemiluminescence intensity is known to be affected by the concentration of organic solvent in the mobile phase (Hayakawa et al., 1991). In view of the fact that the optimum acetonitrile concentration in the mobile phase was $50 \%$ for the elution and separation of 1-, 2-APs and 2-AFR, we examined the optimum concentrations of TCPO and hydrogen peroxide in the chemiluminescence reagent solution. The signal to noise $(\mathrm{S} / \mathrm{N})$ ratio of $1-\mathrm{AP}$ increased with increasing TCPO concentration at concentrations above $0.02 \mathrm{mM}$ and peaked at $0.15 \mathrm{mM}$ TCPO. Furthermore, the S/N ratios of 2-AFR and 2-AP increased with increasing TCPO concentration, peaking at $0.4 \mathrm{mM}$ TCPO. The $\mathrm{S} / \mathrm{N}$ ratios of 2-AFR and 1-AP increased with increasing hydrogen peroxide concentration at concentrations above $4 \mathrm{mM}$ and peaked at $15 \mathrm{mM}$ hydrogen peroxide. On the other hand, the S/N ratios of 2-AP increased with increasing hydrogen peroxide concentration, peaking at $30 \mathrm{mM}$ hydrogen peroxide. In view of the fact that the concentration of 2-NP in airborne particulates is usually lower than the concentrations of 2-NFR and $1-\mathrm{NP}$, the concentrations of chemiluminescence reagents should be optimum to obtain the highest sensitivity of 2-AP. Thus, we used $0.4 \mathrm{mM}$ TCPO and 30 $\mathrm{mM}$ hydrogen peroxide in the following experiments.

Under the above conditions, the detection limits $(\mathrm{S} / \mathrm{N}$ $=3$ ) of 2-NFR, 2-NP and 1-NP by the proposed HPLCCLD system were 4, 10 and $1 \mathrm{fmol}$, respectively. These values were better than the values obtained by the previous system. For example, the previous detection limit of 1-NP was $10 \mathrm{fmol}$ (Tang et al., 2005). The calibration curves were straight in the ranges from $10 \mathrm{fmol}$ to $10 \mathrm{pmol}$ (2-NFR), from $50 \mathrm{fmol}$ to $50 \mathrm{pmol}(2-\mathrm{NP})$ and from $5 \mathrm{fmol}$ to $10 \mathrm{pmol}(1-\mathrm{NP})$, respectively, with correlation coefficients over 0.999 and RSDs less than $5 \%$. 


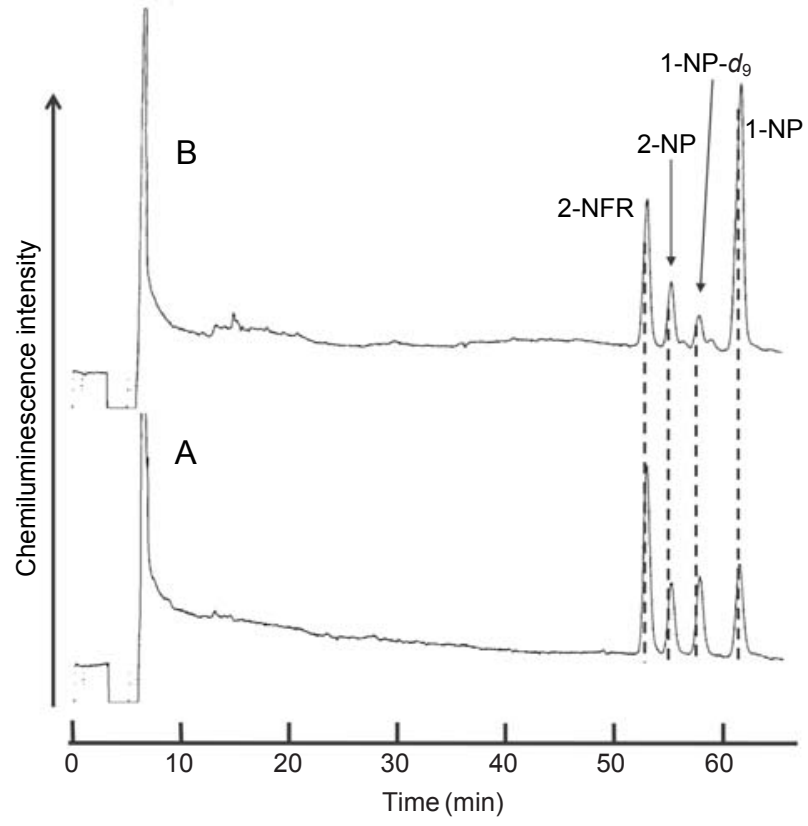

Fig. 2. Typical chromatograms of (A) standard mixture of 1-, 2-NPs, 2-NFR and 1-NP- $d_{9}$ and (B) extracts from airborne particulates collected at the Noto Peninsula. (A) Airborne particulates were collected by a high-volume air sampler at a flow rate of $1,500 \mathrm{~L} \mathrm{~min}^{-1}$ from January 4 to 11,2008 . One-fourth of the filter was used. (B) Injected amounts of 1-, 2-NPs, 2-NFR and $1-\mathrm{NP}-d_{9}$ were $5,50,50$ and $5 \mathrm{fmol}$, respectively.

\section{3 Determination of 1-, 2-NPs and 2-NFR Extracted from Airborne Particulates}

Fig. 2 shows typical chromatograms of (A) the standard mixture of 1-, 2-NPs and 2-NFR and (B) the extracts from airborne particulates collected at Kanazawa University Wajima Air Monitoring Station on the Noto Peninsula, Japan from January 4 to 11,2008. 2-NFR, 2-NP and 1-NP were chemilumigenically determined as their corresponding amino-derivatives in chromatogram B. This result suggests that the proposed HPLCCLD system is suitable for the determination of NPAHs in air samples collected at background monitoring sites. The calculated concentrations of 1-NP, 2-NP and 2NFR were $1.8 \mathrm{fmol}(450 \mathrm{fg}) \mathrm{m}^{-3}, 0.65 \mathrm{fmol}(160 \mathrm{fg})$ $\mathrm{m}^{-3}$ and $6.0 \mathrm{fmol}(1,500 \mathrm{fg}) \mathrm{m}^{-3}$, respectively. The stable baseline of chromatogram A suggests that the proposed system can determine these NPAHs at even much lower concentration.

\subsection{Atmospheric Concentrations of 1- and 2-NPs and 2-NFR at Noto Peninsula}

1-, 2-NPs and 2-NFR in airborne particulates collected in January and July, 2007 at Noto Peninsula were determined by the proposed method. The atmospheric concentrations were not more than sub $\mathrm{pg} \mathrm{m}^{-3}$ levels.
Table 2. Atmospheric concentrations $\left(\mathrm{fg} \mathrm{m}^{-3}\right)^{1)}$ of 1-, 2-NPs and 2-NFR at Noto Peninsula.

\begin{tabular}{lccr}
\hline Month $^{2)}$ & $1-\mathrm{NP}$ & 2-NP & \multicolumn{1}{c}{ 2-NFR } \\
\hline January & $840 \pm 280$ & $230 \pm 100$ & $1900 \pm 520$ \\
July & $80 \pm 33$ & $56 \pm 31$ & $510 \pm 290$ \\
\hline
\end{tabular}

${ }^{1)}$ Each data means mean \pm SD of four different week samples.

${ }^{2}$ Samplings were performed in January and July, 2007. The filter was newly changed every week.

They were compared in Table 2 . The concentrations of 1-, 2-NPs and 2-NFR were higher in winter (January) than in summer (July) by the factors of $1 / 3.7,1 / 10.5$ and $1 / 4.1$, respectively. In both seasons, the concentrations were in decreasing order, $[2-\mathrm{NFR}]>[1-\mathrm{NP}]>[2-$ $\mathrm{NP}$. It has been reported that PAHs exhausted from coal-burning systems especially in winter season in North-East China were long-range transported to Noto Peninsula over the Japan Sea (Yang et al., 2007). The concentration level of 1-NP at Noto Peninsula was much lower than that at Shenyang (annual average concentration $107 \pm 81 \mathrm{pg} \mathrm{m}^{-3}$ (Hattori et al., 2007)) by the factor of 3 or 4 orders of magnitude. This is the first report that the atmospheric concentrations of 1-, 2-NPs and 2-NFR at Noto Peninsula were determined. The proposed method is useful to know the behaviors of trace levels of NPAHs at background monitoring sites such as Noto Peninsula. The long-range transport of NPAHs from Asian Continent to Japan clarified by the proposed method will be reported elsewhere.

\section{CONCLUSIONS}

A HPLC-CLD system has been developed for the determination of trace levels of 1, 2-NPs and 2-NFR in airborne particulates by modifying our previous system. The new system uses a reducer column packed with $\mathrm{Pt} / \mathrm{Rh}$, a chemiluminescence detector and a combination of monomeric and polymeric ODS columns for the separation column. The switching time of the switching valve and the concentrations of TCPO and hydrogen peroxide in the chemiluminescence reagent solution were optimized. The detection limits $(\mathrm{S} / \mathrm{N}=3)$ of 1-, 2-NPs and 2-NFR by the proposed system were 1,10 and $4 \mathrm{fmol}$, respectively. The proposed system determined trace levels of 1-, 2-NPs and 2-NFR in airborne particulates collected at Noto Peninsula. The atmospheric concentrations were not more than pg $\mathrm{m}^{-3}$ levels.

\section{ACKNOWLEDGEMENTS}

This research was supported by a Grant in Aid for 
Scientific Research from the Ministry of Education, Culture, Sports, Science, and Technology, Japan (Nos. 21256001 and 21390034), the Global Environment Research Fund from the Ministry of Environment, Japan (B-0905), Steel Industry Foundation for the Advancement of Environmental Protection Technology and Asia Research Fund from Heiwa Nakajima Foundation. This research was also a Joint Project supported by JSPSKOSEF Japan-Korea Basic Scientific Cooperation Program. We also wish to express our thanks to Professor Akihisa Hirayama (Kyoto Pharmaceutical University, Japan) for his kind gift of 2-NP.

\section{REFERENCES}

Arey, J., Zielinska, B., Atkinson, R., Winer, A.M., Ramdahl, T., Pitts, J.N. Jr. (1986) The formation of nito-PAH from the gas-phase reactions of fluoranthene and pyrene with $\mathrm{OH}$ radical in the presence of NOx. Atmospheric Environment 20, 2339-2345.

Hattori, T., Tang, N., Tamura, K., Hokoda, A., Yang, X.-Y., Igarashi, K., Ohno, M., Okada, Y., Toriba, A., Hayakawa, K. (2007) Profiles of particulate-bound polycyclic aromatic hydrocarbons and their nitrated derivatives in three typical cities, Liaoning Province, China. Environmental Forensics 8(1 \& 2), 165-172.

Hayakawa, K., Kitamura, R., Butoh, M., Imaizumi, N., Miyazaki, M. (1991) Determination of Diamino- and Aminopyrenes by High-Performance Liquid Chromatography with Chemiluminescence Detection. Analytical Sciences 7, 573-577.

Hayakawa, K., Terai, N., Suzuki, K., Dinning, P.G., Yamada, M., Miyazaki, M. (1993) Chromatographic Determination Method for 1-Nitropyrene and Its Metabolites in Biological Samples with Fluorescence Detection after On-line Reduction. Biomedical Chromatography 7, 262-266.

Hayakawa, K., Murahashi, T., Butoh, M., Miyazaki, M. (1995) Determination of 1,3-, 1,6-, and 1,8-Dinitropyrenes and 1-Nitropyrene in Urban Air by High-Performance Liquid Chromatography Using Chemiluminescence Detection. Environmental Science \& Technology 29, 928-932.

Hayakawa, K., Noji, K., Tang, N., Toriba, A., Kizu, R. (2001) A High-Performance Liquid Chromatographic System Equipped with Reducer and Concentrator Columns for Determination of Trace Levels of Nitropolycyclic Aromatic Hydrocarbons in Extracts from DieselEngine Exhaust Particulates. Analytica Chimica Acta
445, 205-212.

Imaizumi, N., Hayakawa, K., Suzuki, Y., Miyazaki, M. (1990) Determination of Nitrated Pyrenes and Their Derivatives by High-Performance Liquid Chromatography with Chemiluminescence Detection after Online Electrochemical Reduction. Noriko Imaizumi, Biomedical Chromatography 4, 108-112.

Kizu, R., Ishii, K., Kobayashi, J., Hashimoto, T., Koh, E., Namiki, M., Hayakawa, K. (2000) Antiandrogenic Effect of Crude Extract of C-Heavy Oil. Materials Science \& Engineering C 12(1-2), 97-102.

Motoyama, Y., Bekki, K., Chung, S.-W., Tang, N., Kameda, T., Toriba, A., Taguchi, K., Hayakawa, K. (2009) Oxidative stress more strongly induced by ortho- than para-quinoid polycyclic aromatic hydrocarbons in A549 cells. Journal of Health Science 55(5), 845-850.

Murahashi, T., Hayakawa, K. (1997) A Sensitive Method for the Determination of 6-Nitrochrysene, 2-Nitrofluoranthene and 1-, 2- and 4-Nitropyrenes in Airborne Particulates Using High-Performance Liquid Chromatography with Chemiluminescence Detection. Analytica Chimica Acta 343, 251-257.

Murahashi, T., Kizu, R., Kakimoto, H., Toriba, A., Hayakawa, K. (1999) 2-Nitrofluoranthene, 1-, 2- and 4-Nitropyrenes and 6-Nitrochrysene in Diesel-Engine Exhaust and Airborne Particulates. Journal of Health Science 45(5), 244-250.

Tang, N., Toriba, A., Kizu, R., Hayakawa, K. (2003) Improvement of an Automatic HPLC System for Nitropolycyclic Aromatic Hydrocarbons: Removal of an Interfering Peak and Increase in the Number of Analytes. Analytical Sciences 19(2), 249-253.

Tang, N., Taga, R., Hattori, T., Toriba, A., Kizu, R., Hayakawa, K. (2005) Simultaneous determination of twentyone mutagenic nitropolycyclic aromatic hydrocarbons by high-performance liquid chromatography with chemiluminescence detection, Proceedings of the $13^{\text {th }}$ International Symposium, Bioluminescence and Chemiluminescence Progress and Perspective, ed. by A. Tsuji et al., World Science, London, pp. 441-444.

Tokiwa, H., Kitamori, S., Takahashi, K., Ohnishi, Y.(1980) Mutagenic and chemical assay of extracts of airborne particulates. Mutation Research 77, 99-108.

Yang, X.-Y., Okada, Y., Tang, N., Matsunaga, S., Tamura, K., Kameda, T., Toriba, A., Hayakawa, K. (2007) Longrange transportation of polycyclic aromatic hydrocarbons from China to Japan. Atmospheric Environment 41(22), 2710-2718.

(Received 18 November 2010, revised 18 February 2011, accepted 10 March 2011) 